To appear in Proc. 48th IEEE CDC

\title{
Backstepping Control Design for Motion Coordination of Self-Propelled Vehicles
}

\author{
Rochelle Mellish and Derek A. Paley
}

\begin{abstract}
Motion coordination of autonomous vehicles has applications from target surveillance to climate monitoring. Previous research has yielded stabilizing control laws for a selfpropelled-vehicle model with first-order rotational dynamics; however, this model may not adequately describe the rotational dynamics of vehicles in the atmosphere or ocean. This paper describes the design of backstepping algorithms for the decentralized control of self-propelled vehicles with secondorder rotational dynamics. We design backstepping controls for planar parallel and circular formations in the absence of a flowfield and in the presence of a steady, uniform flowfield. These controls extend prior results to a more realistic vehicle model.
\end{abstract}

\section{INTRODUCTION}

Research in motion coordination of autonomous vehicles is directly applicable to a number of defense and environmental scenarios, including surveillance [3], [5], [9], wind and temperature measurement for climate monitoring [16], and modeling collective behavior of biological systems [12]. In many applications of coordinated motion, each vehicle in the network is not controlled by a central computer, but rather by a computer on each vehicle. Each agent communicates with its neighbors to automatically control its relative position and orientation [4]. This decentralized communication and control framework enables each group member to act independently and makes the group robust to the failure of an individual agent [8].

Ongoing research in the coordinated motion of autonomous vehicles has focused on the stabilization of planar formations of self-propelled particles in the plane. In prior work, each particle controls the rate of change of the orientation of its velocity; hence, the rotational dynamics are first-order differential equations [14]. The steering control is modeled as a force orthogonal to the particle's velocity so that the particle's direction of travel is under control, but the speed is constant [10]. Formation control laws for this particle model have been developed for parallel and circular formations in the absence of a flowfield [14] and in a uniform, time-invariant flowfield [11]. All-to-all and limited communication frameworks have been considered [14], [15].

Expressing the controllers in terms of shape variables rather than group variables reduces sensing requirements and is possible when the global location of the group is

This work was partially supported by ONR grant N00014-09-1-1058.

D. Paley is an assistant professor in the Department of Aerospace Engineering, University of Maryland College Park, College Park, MD 20742, USA dpaleyeumd. edu

R. Mellish is a graduate student in the Department of Aerospace Engineering, University of Maryland College Park, College Park, MD 20742, USA rnmeumd.edu not related to the goal configuration [17]. For instance, the control laws used to drive the particle models presented in [14] are referred to as shape control laws because the variables that appear in the controllers may be expressed in terms of relative positions and orientations between pairs of particles [6].

In the model of steering control introduced here, the steering control regulates the angular acceleration of the velocity orientation. This level of control is particularly relevant in the context of planar rigid-body motion, where a dynamic vehicle model must account not only for motion of the agent's center of mass, but also for rotational motion about the center of mass. We use this second-order model to derive a control law that stabilizes the velocity orientation of each particle relative to the other particles in a formation. The control design follows the iterative process of integrator backstepping, in which the existing states of the first-order model are recursively used to stabilize steady motions of the second-order model [1], [13].

Recent work in the control of multi-agent systems has incorporated the backstepping control design technique. In [2] backstepping is used to design a controller that will regulate the second-order translational dynamics in order to stabilize a planar formation of three vehicles. The communication framework is modeled as a directed graph, and the goal configuration is a triangular formation. The goal of this paper is different than that of [2] in that we consider parallel and circular formations of self-propelled particles. These two motion primitives can be utilized in theory for an unlimited number of vehicles, and they serve as the basis for more complicated collective motions [14].

The contribution of this paper is to present backstepping control algorithms for the stabilization of parallel and circular formations in a self-propelled-vehicle model with secondorder rotational dynamics. We use this procedure to design stabilizing controls in the absence of a flowfield and then in the presence of a moderate-strength, time-invariant flowfield. In the latter case, we assume the flowfield is known, uniform, and steady. The backstepping control laws retain the shapecontrol characteristics of their first-order counterparts, where the shape-space includes the derivative of the relative orientations. The results of the first-order model can be preserved under the second-order dynamics, as long as each vehicle knows its own turning rate. We illustrate the solutions of the closed-loop system with simulations.

The outline of this paper is as follows. In Section II we give a brief summary of backstepping control design while relating it to the particle model with and without a flowfield. 
In Section III we present a backstepping controller for the flow-free model. In Section IV we repeat the backstepping design for motion coordination in a uniform, time-invariant flowfield. Section V summarizes the paper and describes ongoing research.

\section{PARTICLE MODEL}

To design a backstepping control for planar collective motion, we begin by defining the particle model for particle motion in the absence of a flowfield [14]. Let $r_{k}$ be the position of the $k$ th particle and $\dot{r}_{k}=e^{i \theta_{k}}$ be its (unit) velocity. We have

$$
\begin{aligned}
\dot{r}_{k} & =e^{i \theta_{k}} \\
\dot{\theta}_{k} & =u_{k},
\end{aligned}
$$

where $k=1, \ldots, N$ and $u_{k}$ represents the steering control. We rewrite these equations in real coordinates so that the original states and control now represent the first component of a higher-ordered system, i.e.,

$$
\begin{aligned}
& \dot{\eta}_{1, k}=\cos \eta_{3, k} \\
& \dot{\eta}_{2, k}=\sin \eta_{3, k} \\
& \dot{\eta}_{3, k}=\xi_{k} .
\end{aligned}
$$

The $\eta_{n, k}, n=1, \ldots, 3$ represent the state variables $\operatorname{Re}\left\{r_{k}\right\}$, $\operatorname{Im}\left\{r_{k}\right\}$, and $\theta_{k}$, respectively. $\xi_{k}=u_{k}$ is the state-feedback control, which is expressed in terms of the shape variables $\eta_{3, j}-\eta_{3, k}$ and $\left(r_{k}-r_{j}\right) e^{-i \theta_{k}}$. The higher-ordered system with control $a_{k}$ of the rotational acceleration $\dot{\xi}_{k}=\ddot{\theta}_{k}$ is

$$
\begin{aligned}
\dot{\eta}_{1, k} & =\cos \eta_{3, k} \\
\dot{\eta}_{2, k} & =\sin \eta_{3, k} \\
\dot{\eta}_{\eta_{, k}} & =\xi_{k} \\
\dot{\xi}_{k} & =a_{k} .
\end{aligned}
$$

$a_{k}$ is the control input that we design using backstepping. Similarly to the first-order case, this higher-level controller is expressed in terms of shape variables; we introduce a new shape variable, $\xi_{j}-\xi_{k}$.

When a uniform, time-invariant flowfield is considered, the particle model (1) becomes [11]

$$
\begin{aligned}
\dot{r}_{k} & =e^{i \theta_{k}}+f_{k} \\
\dot{\theta}_{k} & =u_{k} .
\end{aligned}
$$

The flowfield measured at the location of the $k$ th particle is given by $f_{k}$, where $f_{k} \in \mathbb{R}^{2}$. The model (4) can be rewritten as

$$
\begin{aligned}
& \dot{r}_{k}=s_{k} e^{i \gamma_{k}} \\
& \dot{\gamma}_{k}=w_{k} .
\end{aligned}
$$

The variables $s_{k}$ and $\gamma_{k}$ represent the magnitude and orientation of the particle's inertial velocity, respectively, and $w_{k}$ is the control. The variable $s_{k}$ can be computed as

$$
s_{k}=\left|\cos \eta_{3, k}+i \sin \eta_{3, k}+f_{k}\right| .
$$

When the flow $f_{k}$ is oriented along the real axis, then $f_{k}$ becomes $\beta$, where $\beta<1$. In this case, $s_{k}$ becomes [11]

$$
s_{k}=\beta \cos \gamma_{k}+\sqrt{1-\beta^{2} \sin ^{2}\left(\gamma_{k}\right)}>0 .
$$

When a uniform, time-invariant flow field is considered, the model (3) becomes

$$
\begin{aligned}
\dot{\eta}_{1, k} & =\cos \eta_{3, k}+\left\langle f_{k}, 1\right\rangle \\
\dot{\eta}_{2, k} & =\sin \eta_{3, k}+\left\langle f_{k}, i\right\rangle \\
\dot{\eta}_{3, k} & =\xi_{k} \\
\dot{\xi}_{k} & =a_{k} .
\end{aligned}
$$

Similar to the expression we used for (5), we may express (7) in terms of the particle speed, $s_{k}$. Instead of using the $\eta_{n, k}, n=1, \ldots, 3$ to represent the states of the higher-ordered system, as we did in the flow-free case, we now use the variable $\tau_{n, k}$ to represent the orientation of the $k$ th particle's inertial velocity. The control of the higher-ordered system is represented by $\lambda_{k}$, rather than by the variable $a_{k}$ of the flow-free model (3). Thus, the entire higher-ordered system with uniform, time-invariant flow becomes

$$
\begin{aligned}
\dot{\tau}_{1, k} & =s_{k} \cos \tau_{3, k} \\
\dot{\tau}_{2, k} & =s_{k} \sin \tau_{3, k} \\
\dot{\tau}_{3, k} & =\xi_{k} \\
\dot{\xi}_{k} & =\lambda_{k},
\end{aligned}
$$

where $\lambda_{k}$ is the control of the rotational acceleration $\dot{\xi}_{k}=\ddot{\gamma}_{k}$.

We may express model (3) in more general terms as [7]

$$
\begin{aligned}
\dot{\eta}_{k} & =h\left(\eta_{k}\right)+g\left(\eta_{k}\right) \xi_{k} \\
\dot{\xi}_{k} & =x_{k}
\end{aligned}
$$

where

$$
h\left(\eta_{k}\right)=\left[\begin{array}{c}
\cos \eta_{3, k} \\
\sin \eta_{3, k} \\
0
\end{array}\right] \quad \text { and } \quad g\left(\eta_{k}\right)=\left[\begin{array}{l}
0 \\
0 \\
1
\end{array}\right]
$$

Let $\phi_{k}(\eta)$ be the desired control of the $\eta$ dynamics, where $\eta=\left[\eta_{1}, \ldots, \eta_{N}\right]^{T}$. Using the transformation $z_{k}=\xi_{k}-\phi_{k}(\eta)$, (9) may be rewritten as [7]

$$
\begin{aligned}
& \dot{\eta}_{k}=\left[h\left(\eta_{k}\right)+g\left(\eta_{k}\right) \phi_{k}(\eta)\right]+g\left(\eta_{k}\right) z_{k} \\
& \dot{z}_{k}=v_{k}
\end{aligned}
$$

where $v_{k}=a_{k}-\dot{\phi}_{k}$ is the backstepping control. In this higherordered model (10), the control of the subsystem $\phi_{k}(\eta)$ is now treated as a state, while the variable $z_{k}$ represents the difference between the actual controller and the desired controller of the lower-ordered system [13]. Model (8) can be expressed similarly.

\section{FLOW-FREE MOTION COORDINATION}

We now describe a backstepping control design for the flow-free particle model in order to achieve asymptotic convergence to either a synchronized or a circular formation. Phase synchronization is attained when the average linear momentum of the collective motion is maximized, that is, when the phase angles $\eta_{3, k}=\eta_{3, j}$ for all pairs $j$ and $k$ [14]. On the other hand, if each particle in model (3) is driven in a circular trajectory of radius $1 /\left|\omega_{0}\right|$ by the control $\dot{\eta}_{3, k}=\omega_{0}$, group circular motion occurs when the centers of each particle's trajectory coincide [14]. 


\section{A. Phase Stabilization}

Consider the model (3) with $\dot{\eta}_{3, k}=\phi_{1, k}(\eta)$ and

$$
\phi_{1, k}(\eta)=-K\left\langle p_{\theta}, i e^{i \eta_{3, k}}\right\rangle .
$$

Equation (11) is a gradient control law for phase stabilization [14]. Assuming unit-mass particles, the average linear momentum is

$$
p_{\theta} \triangleq \frac{1}{N} \sum_{j=1}^{N} e^{i \eta_{3, j}} .
$$

The closed-loop behavior of the $\eta$ dynamics with control $\phi_{1, k}(\eta)$ is established using the Lyapunov function [14]

$$
V_{1}(\eta)=\frac{1}{2}\left\|p_{\theta}\right\|^{2} .
$$

Taking the time derivative of $V_{1}(\eta)$, we obtain

$$
\dot{V}_{1}=\sum_{k=1}^{N} \frac{\partial V_{1}}{\partial \eta_{3, k}} \dot{\eta}_{3, k}=\sum_{k=1}^{N}\left\langle p_{\theta}, i e^{i \eta_{3, k}}\right\rangle \dot{\eta}_{3, k} .
$$

Substituting $\phi_{1, k}(\eta)$ into (13) yields

$$
\dot{V}_{1}=-K \sum_{k=1}^{N}\left\langle p_{\theta}, i e^{i \eta_{3, k}}\right\rangle^{2} \leq 0 .
$$

According to [14, Theorem 1] the potential $V_{1}(\eta)=$ $\frac{1}{2}\left\|p_{\theta}\right\|^{2}$ reaches its unique minimum when $p_{\theta}=0$ (balancing) and its unique maximum when all phases are identical (synchronization). All other critical points of $V_{1}$ are isolated in the reduced space of relative phases (shape space) and are saddle points of $V_{1}$. We are interested in stabilizing the set of synchronized critical points in the model (3), which are attained for the closed-loop $\eta$ dynamics when $K<0$.

Now we design a backstepping control for the higherordered system (3). We use the composite Lyapunov function

$$
V_{1, c}(\eta, z)=V_{1}(\eta)+\frac{1}{2} \sum_{k=1}^{N} z_{k}^{2},
$$

where $z_{k}=\xi_{k}-\phi_{1, k}(\eta)$, and $\phi_{1, k}(\eta)$ is given by (11). The time derivative of $V_{1, c}$ is

$$
\dot{V}_{1, c}=\sum_{k=1}^{N}\left\langle p_{\theta}, i e^{i \eta_{3, k}}\right\rangle \dot{\eta}_{3, k}+z_{k} \dot{z}_{k} .
$$

Substituting $\dot{z}_{k}=v_{k}$ and $\dot{\eta}_{3, k}=\phi_{1, k}(\eta)+z_{k}$ into (15) yields

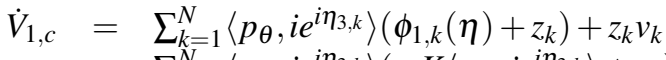

$$
\begin{aligned}
& =\sum_{k=1}^{N}\left\langle p_{\theta}, i e^{i \eta_{3, k}}\right\rangle\left(-K\left\langle p_{\theta}, i e^{i \eta_{3, k}}\right\rangle+z_{k}\right)+z_{k} v_{k} \text {. }
\end{aligned}
$$

Choosing

$$
v_{k}=-\left\langle p_{\theta}, i e^{i \eta_{3, k}}\right\rangle-\kappa z_{k}, \kappa>0
$$

gives

$$
\dot{V}_{1, c}=\sum_{k=1}^{N}-K\left\langle p_{\theta}, i e^{i \eta_{3, k}}\right\rangle^{2}-\kappa z_{k}^{2} \leq 0 .
$$

The control $a_{k}=v_{k}+\dot{\phi}_{1, k}$ that asymptotically stabilizes parallel formations in the model (3) is

$$
\begin{aligned}
& a_{k}=-\left\langle p_{\theta}, i e^{i \eta_{3, k}}\right\rangle-\kappa\left(\xi_{k}+K\left\langle p_{\theta}, i e^{i \eta_{3, k}}\right\rangle\right) \\
& -\frac{K}{N} \sum_{j=1}^{N}\left[\left\langlee^{i \eta_{3, j}}, e^{\left.\left.i \eta_{3, k}\right\rangle\left(\xi_{j}-\xi_{k}\right)\right],}\right.\right.
\end{aligned}
$$

where $\dot{\phi}_{1, k}$ is given by the derivative of (11) and $K<0$.

Theorem 1: Consider the particle model (3) with the backstepping control (17). Under this control, the set of parallel formations where $\eta_{3, k}=\eta_{3, j}$ for all pairs $j$ and $k$ is asymptotically stable when $K<0$.

Proof: $V_{1, c}$ is a positive semi-definite smooth potential. By the invariance principle, we know that the solutions of (3) with the control (11) converge to the largest invariant set $\Lambda$ for which $\dot{V}_{1, c} \equiv 0$. For parallel motion, the largest invariant set is given by

$$
\Lambda=\left\{\left\langle p_{\theta}, i e^{i \eta_{3, k}}\right\rangle=0, z_{k}=0 \forall k\right\} .
$$

The condition that $\left\langle p_{\theta}, i e^{i \eta_{3, k}}\right\rangle=0$ implies $\Lambda$ contains parallel, balanced, and unbalanced motions; only parallel are stable for $K<0$ [14]. $z_{k}=0$ implies $\xi_{k}=\phi_{1, k}(\eta)$; however, from (11) we know that $\phi_{1, k}(\eta)=0$ in $\Lambda$. This implies that $\eta_{3, k}$ is constant for all $k$.

This result is illustrated in Fig. 1, using $N=16, K=-1$, and $\kappa=5$.

\section{B. Stabilization of Circular Formations}

For the stabilization of circular formations, we again consider the model (3) with $\dot{\eta}_{3, k}=\phi_{2, k}(\eta)$ and

$$
\phi_{2, k}(\eta)=\omega_{0}+\omega_{0} K\left\langle P_{k} \mathbf{c}, e^{i \eta_{3, k}}\right\rangle .
$$

Equation (18) represents a decentralized control law for the $\eta$ dynamics that asymptotically stabilizes the set of circular formations [14]. The $N \times 1$ matrix c contains the centers $c_{k}$, $k=1, \ldots, N$, of the circular paths followed by each of the particles $k$, where

$$
c_{k}=r_{k}+i \omega_{0}^{-1} e^{i \eta_{3, k}} .
$$

$P=I_{N \times N}-\frac{1}{N} \mathbf{1 1}^{T}$ is an $N \times N$ matrix that projects onto the space orthogonal to $\mathbf{1}=[1, \ldots, 1]^{T} \in \mathbb{R}^{N}$ and $P_{k}$ represents the $k$ th row of $P$.

As in the case of synchronized motion, we begin our derivation of the circular formation control law by briefly reviewing the methods used by [14] to analyze the $\eta$ dynamics. Consider the Lyapunov function [14]

$$
V_{2}(\eta)=\frac{1}{2}\langle\mathbf{c}, P \mathbf{c}\rangle
$$

whose derivative is

$$
\dot{V}_{2}=\sum_{k=1}^{N}\left\langle\dot{c}_{k}, P_{k} \mathbf{c}\right\rangle=\sum_{k=1}^{N}\left\langle e^{i \eta_{3, k}}, P_{k} \mathbf{c}\right\rangle\left(1-\omega_{0}^{-1} \dot{\eta}_{3, k}\right) .
$$

If $\phi_{2, k}(\eta)$ is chosen to be the control (18) then the derivative of the Lyapunov function becomes

$$
\dot{V}_{2}=-K \sum_{k=1}^{N}\left\langle P_{k} \mathbf{c}, e^{i \eta_{3, k}}\right\rangle^{2} \leq 0 .
$$

By [14, Theorem 2] we know that the control $\phi_{2, k}(\eta)$ with $K>0$ forces all solutions of the $\eta$ dynamics to converge to the largest invariant set $\Lambda$, where

$$
\left\langle P_{k} \mathbf{c}, e^{i \eta_{3, k}}\right\rangle \equiv 0 \forall k .
$$

In $\Lambda, \dot{\eta}_{3, k}=\omega_{0}$ and $\dot{c}_{k}=0$. The condition in (21) is met only when $P \mathbf{c}=0$, which implies $c_{k}=c_{j}$ for all pairs $j$ and $k$.

We use the Lyapunov function (20) to form the composite Lyapunov function, $V_{2, c}=\frac{1}{2}\langle\mathbf{c}, P \mathbf{c}\rangle+\frac{1}{2} \sum_{k=1}^{N} z_{k}^{2}$. Taking the time-derivative along the solutions of (3), we obtain

$$
\dot{V}_{2, c}=\sum_{k=1}^{N}\left\langle e^{i \eta_{3, k},}, P_{k} \mathbf{c}\right\rangle\left(1-\omega_{0}^{-1} \dot{\eta}_{3, k}\right)+z_{k} \dot{z}_{k} .
$$



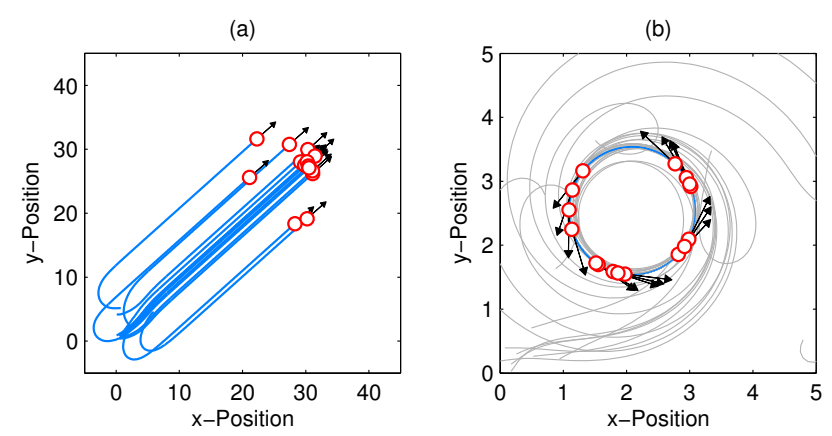

Fig. 1. (a) Synchronized motion of self-propelled particles with secondorder rotational dynamics. (b) Stabilization of a circular formation of selfpropelled particles with second-order rotational dynamics

Using $\dot{z}_{k}=v_{k}$ and $\dot{\eta}_{3, k}=\phi_{2, k}(\eta)+z_{k}, \dot{V}_{2, c}$ becomes

$$
\begin{aligned}
& \dot{V}_{2, c}=\sum_{k=1}^{N}\left\langle e^{i \eta_{3, k}}, P_{k} \mathbf{c}\right\rangle\left(1-\omega_{0}^{-1}\left(\phi_{2, k}(\eta)+z_{k}\right)\right)+z_{k} v_{k} \\
& =\sum_{k=1}^{N}-K\left\langle P_{k} \mathbf{c}, e^{i \eta_{3, k}}\right\rangle^{2}-\omega_{0}^{-1}\left\langle P_{k} \mathbf{c}, e^{i \eta_{3, k}}\right\rangle z_{k}+z_{k} v_{k} \text {. }
\end{aligned}
$$

Choosing

$$
v_{k}=-\kappa z_{k}+\omega_{0}^{-1}\left\langle P_{k} \mathbf{c}, e^{i \eta_{3, k}}\right\rangle
$$

yields

$$
\dot{V}_{2, c}=\sum_{k=1}^{N}-K\left\langle P_{k} \mathbf{c}, e^{i \eta_{3, k}}\right\rangle^{2}-\kappa z_{k}^{2} \leq 0 .
$$

If we use the transformation $a_{k}=v_{k}+\dot{\phi}_{2, k}$ and we define the quantity $\tilde{r}_{k}=r_{k}-\frac{1}{N} \sum_{j=1}^{N} r_{j}$, then we can write the secondorder controller for circular motion as

$$
\begin{aligned}
a_{k}= & -\kappa\left(\xi_{k}-\phi_{2, k}(\eta)\right)+\omega_{0} K \xi_{k}\left\langle\tilde{r}_{k}, i e^{i \eta_{3, k}}\right\rangle+K\left(\omega_{0}-\right. \\
& \left.\frac{1}{N} \sum_{j=1}^{N}\left[\left\langle e^{i \eta_{3, j}}, e^{i \eta_{3, k}}\right\rangle\left(\omega_{0}-\left(\xi_{j}-\xi_{k}\right)\right)\right]\right) \\
& +\omega_{0}^{-1}\left(\left\langle\tilde{r}_{k}, e^{i \eta_{3, k}}\right\rangle-\omega_{0}^{-1} \frac{1}{N} \sum_{j=1}^{N}\left\langle i e^{i \eta_{3, j}}, e^{i \eta_{3, k}}\right\rangle\right) .
\end{aligned}
$$

Theorem 2: Consider the particle model (3) with the backstepping control (22). All solutions converge to the set of circular formations with radius $1 /\left|\omega_{0}\right|$ and the direction of rotation determined by the sign of $\omega_{0}$.

Proof: By the invariance principle, we know that the solutions of (3) with the control (18) converge to the largest invariant set $\Lambda$ for which $\dot{V}_{2, c} \equiv 0$. For circular motion, the largest invariant set is given by

$$
\Lambda=\left\{\left\langle P_{k} \mathbf{c}, e^{i \eta_{3, k}}\right\rangle=0, z_{k}=0 \quad \forall k\right\} .
$$

$\left\langle P_{k} \mathbf{c}, e^{i \eta_{3, k}}\right\rangle=0$ implies that $P_{k} \mathbf{c} \equiv 0$, which is only true when all circular centers are the same; that is, $P_{k} \mathbf{c}=0$ if and only if $\mathbf{c}$ is in the span of $\mathbf{1}$. Using (18) along with the fact that $\left\langle P_{k} \mathbf{c}, e^{i \eta_{3, k}}\right\rangle=0$, we have $\phi_{2, k}(\eta)=\omega_{0}$. Thus, all $N$ particles travel around the same circle of radius $1 /\left|\omega_{0}\right| . z_{k}=0$ implies that $\dot{\eta}_{3, k}=\omega_{0}$.

This result is illustrated in Fig. 1 , where $N=16, K=1$, $\kappa=5$, and $\omega_{0}=1$.

\section{COLLECTIVE MOTION IN A FLOWFIELD}

We now design a backstepping control considering a uniform, time-invariant flowfield. In a flowfield, phase synchronization is attained when the inertial phase angles satisfy $\tau_{3, k}=\tau_{3, j}$ for all pairs $j$ and $k$. Group circular motion occurs under the control $\dot{\tau}_{3, k}=\omega_{0} s_{k}$, when the centers of all particle trajectories coincide [11]. We require that each vehicle know the local flowfield.

\section{A. Phase Stabilization in a Uniform Flow}

The model for a particle traveling in a uniform, timeinvariant flowfield is given by (8), where $\dot{\tau}_{3, k}=\phi_{3, k}(\tau)$. We show that the set of parallel formations is stabilized by the control [11]

$$
\phi_{3, k}(\tau)=-K \sum_{k=1}^{N}\left\langle p_{\gamma}, i e^{i \tau_{3, k}}\right\rangle,
$$

where $K<0$. This is proven using the Lyapunov function

$$
V_{3}(\tau)=\frac{1}{2}\left\|p_{\gamma}\right\|^{2}
$$

which we seek to maximize in order to achieve phase synchronization. Similarly to $p_{\theta}$ in the flow-free case, $p_{\gamma}$ is defined as the average inertial linear momentum, i.e.,

$$
p_{\gamma}=\frac{1}{N} \sum_{j=1}^{N} e^{i \tau_{3, j}}
$$

The time-derivative of the Lyapunov function (25) is

$$
\dot{V}_{3}(\tau)=\sum_{k=1}^{N} \frac{\partial V_{3}}{\partial \tau_{3, k}} \dot{\tau}_{3, k}=\sum_{k=1}^{N}\left\langle p_{\gamma}, i e^{i \tau_{3, k}}\right\rangle \phi_{k}(\tau) .
$$

With $\phi_{3, k}(\tau)$ given by 24 , the derivative of the Lyapunov function becomes

$$
\dot{V}_{3}(\tau)=\sum_{k=1}^{N}-K\left\langle p_{\gamma}, i e^{i \tau_{3, k}}\right\rangle^{2} \leq 0 .
$$

From [11, Theorem 1] we know that all solutions converge to the critical set of $V_{3}$. With $K<0$, the set of synchronized motions are asymptotically stable and every other equilibrium is unstable.

We use this result to derive the phase stabilization control law for the higher-ordered system. The composite Lyapunov function is

$$
V_{3, c}(\tau, z)=V_{3}(\tau)+\sum_{k=1}^{N} \frac{1}{2} z_{k}^{2},
$$

where $z_{k}=\xi_{k}-\phi_{3, k}(\tau)$. The time derivative of the composite Lyapunov function is

$$
\dot{V}_{3, c}=\sum_{k=1}^{N}\left\langle p_{\gamma}, i e^{i \tau_{3, k}}\right\rangle \dot{\tau}_{3, k}+z_{k} \dot{z}_{k} .
$$

Using $\dot{z}_{k}=v_{k}$ and $\dot{\tau}_{3, k}=\phi_{3, k}(z)+z_{k}$, the derivative of the composite Lyapunov function becomes

$$
\begin{aligned}
\dot{V}_{3, c} & =\sum_{k=1}^{N}\left\langle p_{\gamma}, i e^{\left.i \tau_{3, k}\right\rangle}\left(\phi_{k}(\tau)+z_{k}\right)+z_{k} v_{k}\right. \\
& =\sum_{k=1}^{N}\left\langle p_{\gamma}, i e^{\left.i \tau_{3, k}\right\rangle}\left(-K\left\langle p_{\gamma}, i e^{i \tau_{3, k}}\right\rangle+z_{k}\right)+z_{k} v_{k} .\right.
\end{aligned}
$$

Choosing

$$
v_{k}=-\left\langle p_{\gamma}, i e^{i \tau_{3, k}}\right\rangle-\kappa z_{k}
$$

gives

$$
\dot{V}_{3, c}=\sum_{k=1}^{N}-K\left\langle p_{\gamma}, i e^{i \tau_{3, k}}\right\rangle^{2}-\kappa z_{k}^{2} \leq 0
$$


Using the transformation $\lambda_{k}=v_{k}+\dot{\phi}_{k}$, the control may be written as

$$
\begin{aligned}
\lambda_{k}= & -\left\langle p_{\gamma}, i e^{i \tau_{3, k}}\right\rangle-\kappa\left(\xi_{k}+K\left\langle p_{\gamma}, i e^{i \tau_{3, k}}\right\rangle\right) \\
& -\frac{K}{N} \sum_{j=1}^{N}\left[\left\langle e^{i \tau_{3, j}}, e^{i \tau_{3, k}}\right\rangle\left(\xi_{j}-\xi_{k}\right)\right] .
\end{aligned}
$$

Theorem 3: Consider the particle model (8) with the backstepping control (26) and flow $f_{k}=\beta<1$. For $K<0$ the set of parallel formations where $\tau_{3, k}=\tau_{3, j}$ for all pairs $j$ and $k$ is asymptotically stable.

The proof for parallel motion in the presence of a timeinvariant flowfield follows the proof given for Theorem 1, with $\eta_{3, k}$ replaced by $\tau_{3, k}$. This result is illustrated in Fig. 2, using $N=16, K=-1, \kappa=5$.

\section{B. Stabilization of Circular Formations in a Uniform Flow}

For collective motion control of circular formations in a time-invariant flowfield, we consider the model (8) with $\dot{\tau}_{3, k}=\phi_{4, k}(\tau)$, where

$$
\phi_{4, k}(\tau)=\omega_{0}\left(s_{k}+K\left\langle P_{k} \mathbf{c}, e^{i \tau_{3, k}}\right\rangle\right)
$$

and $K>0$. Similarly to the flow-free case, the center of each particle's trajectory is given by $c_{k}=r_{k}+i \omega_{0}^{-1} e^{i \tau_{3, k}}$, and the radius of the circular trajectory is given by $1 /\left|\omega_{0}\right|[11]$. We reiterate the stability analysis of [11] to show that the spacing control $\phi_{4, k}(\tau)$ asymptotically stabilizes the set of circular formations. This is proven using the Lyapunov function

$$
V_{4}(\tau)=\frac{1}{2}\langle\mathbf{c}, P \mathbf{c}\rangle,
$$

which has the time derivative

$$
\begin{aligned}
\dot{V}_{4} & =\sum_{k=1}^{N}\left\langle\dot{c}_{k}, P_{k} \mathbf{c}\right\rangle \\
& =\sum_{k=1}^{N}\left\langle P_{k} \mathbf{c}, e^{i \tau_{3, k}}\right\rangle\left(s_{k}-\omega_{0}^{-1} \phi_{4, k}(\tau)\right) .
\end{aligned}
$$

Substituting $\phi_{4, k}(\tau)$ from (27) gives

$$
\dot{V}_{4}=-\sum_{k=1}^{N} K\left\langle P_{k} \mathbf{c}, e^{i \tau_{3, k}}\right\rangle^{2} \leq 0 .
$$

As stated in [11, Theorem 3], the control (27) forces the convergence of all solutions of the $\tau$ dynamics in (8) to the largest invariant set $\Lambda$ of $V_{4}$, in which

$$
\left\langle P_{k} \mathbf{c}, e^{i \tau_{3, k}}\right\rangle \equiv 0
$$

In $\Lambda, \dot{\tau}_{3, k}=\omega_{0} s_{k}$ and $\dot{c}_{k}=0$. Therefore, the condition (29) is met only when $P \mathbf{c}=0$, which implies that $c_{k}=c_{j}$ for all pairs $j$ and $k$.

Using (28) we form the composite Lyapunov function

$$
V_{4, c}(\tau, z)=V_{4}(\tau)+\frac{1}{2} \sum_{k=1}^{N} z_{k}^{2}
$$

whose derivative along solutions of (8) is

$$
\dot{V}_{4, c}=\sum_{k=1}^{N}\left\langle P_{k} \mathbf{c}, e^{i \tau_{3, k}}\right\rangle\left(s_{k}-\omega_{0}^{-1} \dot{\tau}_{3, k}\right)+z_{k} \dot{z}_{k} .
$$

Making the substitutions $\dot{z}_{k}=v_{k}$ and $\dot{\tau}_{3, k}=\phi_{4, k}(\tau)+z_{k}$, we rewrite the time derivative as

$$
\begin{aligned}
\dot{V}_{4, c} & =\sum_{k=1}^{N}\left\langle P_{k} \mathbf{c}, e^{i \tau_{3, k}}\right\rangle\left(s_{k}-\omega_{0}^{-1}\left(\phi_{k}(\tau)+z_{k}\right)\right)+z_{k} v_{k} \\
& =\sum_{k=1}^{N}-K\left\langle P_{k} \mathbf{c}, e^{i \tau_{3, k}}\right\rangle^{2}-\omega_{0}^{-1}\left\langle P_{k} \mathbf{c}, e^{i \tau_{3, k}}\right\rangle z_{k}+z_{k} v_{k} .
\end{aligned}
$$

Choosing the control $v_{k}$ to be

$$
v_{k}=-\kappa z_{k}+\omega_{0}^{-1}\left\langle P_{k} \mathbf{c}, e^{i \tau_{3, k}}\right\rangle
$$

(a)

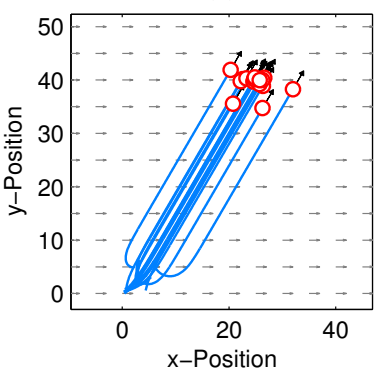

(b)

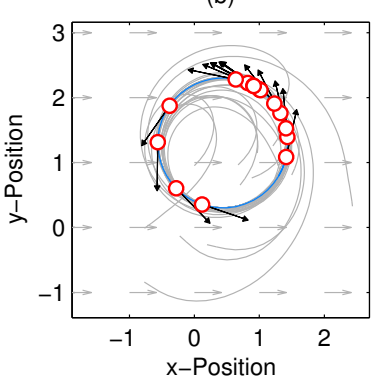

Fig. 2. (a) Stabilization of a parallel formation in a uniform, time-invariant flowfield and (b) stabilization of a circular formation in a uniform, timeinvariant flowfield; $\beta=0.5$ in both cases.

yields

$$
\dot{V}_{4, c}=\sum_{k=1}^{N}-K\left\langle P_{k} \mathbf{c}, e^{i \tau_{3, k}}\right\rangle^{2}-\kappa z_{k}^{2} \leq 0 .
$$

The control may be transformed into $\lambda_{k}$ using the transformation $\lambda_{k}=v_{k}+\dot{\phi}_{4, k}$. Thus,

$$
\begin{aligned}
\lambda_{k}= & -\kappa\left(\xi_{k}-\phi_{4, k}(\tau)\right)+\omega_{0} K \xi_{k}\left\langle\tilde{r}_{k}, i e^{i \tau_{3, k}}\right\rangle+K\left(\omega_{0} s_{k}-\right. \\
& \left.\frac{1}{N} \sum_{j=1}^{N}\left[\left\langle e^{i \tau_{3, j}}, e^{i \tau_{3, k}}\right\rangle\left(\omega_{0} s_{j}-\left(\xi_{j}-\xi_{k}\right)\right)\right]\right) \\
& +\omega_{0}^{-1}\left(\left\langle\tilde{r}_{k}, e^{i \tau_{3, k}}\right\rangle-\omega_{0}^{-1} \frac{1}{N} \sum_{j=1}^{N}\left\langle i e^{i \tau_{3, j}}, e^{\left.i \tau_{3, k}\right\rangle}\right)\right. \\
& +\omega_{0} \dot{s}_{k}
\end{aligned}
$$

where $\phi_{4, k}(\tau)$ is given by (27) and

$$
\dot{s}_{k}=-\beta \sin \tau_{3, k}\left[1+\frac{\beta \cos \tau_{3, k}}{\sqrt{1-\beta^{2} \sin ^{2}\left(\tau_{3, k}\right)}}\right] \xi_{k} .
$$

Theorem 4: Consider the particle model (8) with the backstepping control (31) and flow $f_{k}=\beta<1$. All solutions converge to the set of circular formations of radius $1 /\left|\omega_{0}\right|$ and direction of rotation determined by the sign of $\omega_{0}$.

Proof: By the invariance principle, we know that the solutions of (3) with the control (27) converge to the largest invariant set $\Lambda$ for which $\dot{V}_{4, c} \equiv 0$. For circular motion in a uniform, time-invariant flowfield, the largest invariant set is given by

$$
\Lambda=\left\{\left\langle P_{k} \mathbf{c}, e^{i \tau_{3, k}}\right\rangle=0, z_{k}=0 \forall k\right\}
$$

The first condition implies that $P_{k} \mathbf{c} \equiv 0$, which is only true when all circular centers are the same. The second condition implies that $\xi_{k}=\phi_{4, k}(\tau)$; thus, we have $\xi_{k}=\phi_{4, k}(\tau)=\omega_{0} s_{k}$.

This result is illustrated in Fig. 2, with $N=16, K=1$, $\kappa=5, \omega_{0}=1$.

\section{CONCLUSION}

This paper proposes a backstepping control design for the stabilization of formations of $N$ self-propelled particles with second-order rotational dynamics. Stabilization of the higherordered system relies on the assumed stability of the original system, as presented in [14] and [11]. In exploiting the Lyapunov functions used to prove the stability of formations with first-order rotational dynamics, we construct composite 
Lyapunov functions that are used to design controls to stabilize formations in the higher-ordered system.

There are certain requirements that each vehicle must meet. The first requirement is that each vehicle must know the local flowfield. The second requirement is the result of extending the first-order models to include second-order dynamics: doing so introduces the new sensing requirement that each vehicle know its angular velocity. Extensions of this backstepping algorithm include extending the models (3) and (7) to include control of second-order translational dynamics. Experiments on a multi-vehicle testbed are planned to verify the efficacy of the backstepping control laws derived in this paper.

\section{REFERENCES}

[1] I. Ali, G. Radice, and J. Kim. Backstepping control design with actuator torque bound for spacecraft attitude maneuver. Journal of Guidance, Control, and Dynamics, 33(1):254-259, January-February 2010.

[2] Y.Y. Chen and Y. Tian. A backstepping design for directed formation control of three-coleader agents in the plane. International Journal of Robust and Nonlinear Control, 19(7):729-745, 2008.

[3] C. Chong and S. P. Kumar. Sensor networks: Evolution, opportunities, and challenges. In Proceedings of the IEEE, volume 91, pages 12471256, August 2003.

[4] P. Ferguson and J. How. Decentralized estimation algorithms for formation flying spacecraft. In AIAA Guidance, Navigation, and Control Conference and Exhibit, Austin, Texas, August 2003.

[5] E.W. Frew, D.A. Lawrence, and S. Morris. Coordinated standoff tracking of moving targets using Lyapunov guidance vector fields. J. Guidance, Control, and Dynamics, 31(2):290-306, 2008.
[6] E.W. Justh and P.S. Krishnaprasad. Equilibria and steering laws for planar formations. Systems and Control Letters, 52(1):25-38, May 2004.

[7] H. Khalil. Nonlinear Systems. Prentice Hall, Upper Saddle River, NJ 07458, 3rd. edition, 2002.

[8] A. Makarenko and H.F. Durrant-Whyte. Decentralized data fusion and control in active sensor networks. In International Conference on Information Fusion, Stockholm, Sweden, 2004.

[9] J.A. Marshall, M.E. Broucke, and B.A. Francis. Formations of vehicles in cyclic pursuit. IEEE Trans. Automatic Control, 49(11):1963-1974, 2004.

[10] N. Moshtagh and A. Jadbabaie. Steering laws for distributed motion coordination of kinematic agents in three dimensions. 47th IEEE Conference on Decision and Control, pages 1741-1746, Cancun, Mexico, December 2008.

[11] D. Paley and C. Peterson. Stabilization of collective motion in a time-invariant flowfield. Journal of Guidance, Control, and Dynamics, 32(3):771-779, May-June 2009.

[12] C.W. Reynolds. Flocks, herds, and schools: A distributed behavioral model. In Computer Graphics, volume 21 of SIGGRAPH '87 Conference Proceedings, pages 25-34, 1987.

[13] R. Sepulchre, M. Jankovic, and P.V. Kokotovic. Constructive Nonlinear Control. Springer, New York, 1997.

[14] R. Sepulchre, D. Paley, and N. Leonard. Stabilization of planar collective motion: All-to-all communication. IEEE Transactions on Automatic Control, 52(5):811-824, May 2007.

[15] R. Sepulchre, D. A. Paley, and N. E. Leonard. Stabilization of planar collective motion with limited communication. IEEE Transactions on Automatic Control, 53(3):706-719, April 2008.

[16] L. Techy, D. A. Paley, and C. A. Woolsey. UAV coordination on convex curves in wind: An environmental sampling application. 2009 European Control Conference, pages 4967-4972, Budapest, Hungary, August 2009.

[17] F. Zhang, M. Goldgeier, and P.S. Krishnaprasad. Control of small formations using shape coordinates. volume 2, pages 2510-2515, Taipei, Taiwan, September 2003. International Conference on Robotics and Automation. 